\title{
Seeing is believing
}

\section{Peering down an eyepiece is becoming a thing of the past. Tim Chapman takes a look into the digital world of a new generation of microscopes and imaging systems.}

\begin{abstract}
f anything has remained constant over centuries of biological research, it is the value of the evidence of your own eyes, gained by examining specimens - living or dead — in as much detail as possible. Today's microscopes are, of course, a world away from the optical systems used by the pioneers of biology. Researchers in genomics and proteomics need to study molecular interactions within living cells in detail, whereas the high-throughput screening required by the pharmaceutical industry demands the automated scanning and analysis of large numbers of samples.
\end{abstract}

\section{Going digital}

Even for the simplest applications, microscopes have embraced digital technology for image analysis and, increasingly, for presenting real-time images without the operator needing to peer down eyepieces. "No company is selling a lot of film-based cameras any more," says Rainer Wegerhoff, section manager at the microscopy training facility run by microscope manufacturers Olympus in Hamburg, Germany. "Everything is going into the digital market because there is so much more benefit for users to use digital imaging instead of film-based imaging."

Digital imaging can be particularly helpful in bioscience applications involving fluorescence. "If you see fluorescence, it's very hard to get good quantifiable image data from a film-based system," Wegerhoff notes. "Most people would start with digital imaging systems. You have only a few milliseconds, so you can resolve single-molecule detection or things that were not possible with video or film."

Although most digital microscopes resemble traditional optical systems with an added facility to display the image digitally, there is now a new generation of innovative microscopes for exclusively digital use, which take the image directly to a computer screen. Olympus's MIC-D is a relatively simple low-power digital microscope designed for education and basic laboratory applications. It has no eyepiece, but plugs into a desktop or laptop computer through a USB connection. Its base houses a low-power CMOS (complementary metal oxide semiconductor) detector, while a swivelling lamp arm with a solid-state LED lamp allows the sample to be illuminated from a range of angles, and in various illumination modes.
The CMOS detector produces an image of just $640 \times 480$ pixels, less than a tenth of many laboratory systems, and can be used to look, for example, at pond life and other small creatures, and the general cellular structure of fixed plant tissues. "It's not comparable to real research microscopes; it's an instrument that can be transported from one location to another and used in the field," Wegerhoff says. Several police forces have shown interest in the MIC-D for initial forensic imaging at crime scenes.

Many applications do require higher resolutions, particularly in the medical market. Pathologists, who have traditionally made diagnoses from images recorded on silver halide film, are beginning to use digital images now that charge-coupled devices (CCDs) offering similar resolution to film are more widely available.

The Coolscope system from Nikon, based in Kanagawa, Japan, is specifically targeted at the pathology market. It is based on a 5-megapixel CCD, similar to that used in Nikon's general-purpose DS-5M digital microscope. But the Coolscope combines the microscope, CCD camera and network functions into an integrated tower unit that

\section{LIGHTING UP THE BODY}

The ability to track the molecular action of disease or other biological processes within live subjects is a potentially invaluable tool for studying the broad array of drug targets identified by genomic and proteomic research.

Medical imaging techniques such as magnetic resonance imaging (MRI) and positron emission tomography (PET) can be deployed, but they demand expensive equipment beyond the reach of many labs and provide limited functional information. A simple but effective alternative is to use optical imaging techniques that exploit the properties of light-emitting enzymes.

This approach was pioneered at Stanford University in the mid-1990s by husband-and-wife team Christopher and Pamela Contag, together with David Benaron. The Stanford team infected mice with bacterial pathogens that had been engineered to carry the gene for luciferase, the enzyme that makes fireflies glow. As these pathogens spread through the mice, light emitted by the luciferase could be detected with a highly sensitive CCD camera.

In 1997, Pamela Contag left Stanford to form the company Xenogen to commercialize the technology. Based in Alameda, California, Xenogen now produces both the sensitive light recorders and software required for what it calls biophotonic imaging, and a family of transgenic mice and rats

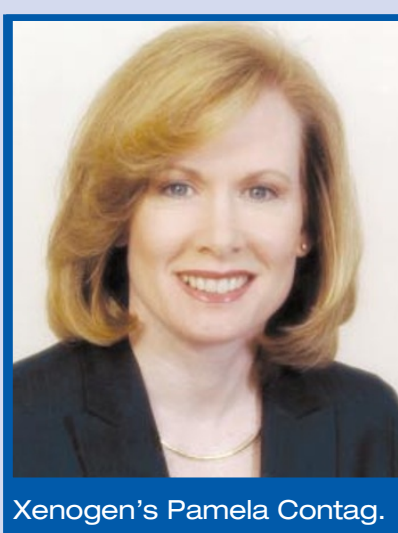

engineered to emit light when tagged genes are activated. Because all imaging is in vivo, the technology allows longitudinal study design. "You start and end the experiment with the same animal and monitor the same animal over time - this allows you to use $60-80 \%$ fewer animals," Contag notes. "That allows experiments to be run at more reasonable cost, and because we're looking at very early in the disease process, these disease models are less stressful for the animals."

The company has now signed licensing deals with a string of pharmaceutical companies including AstraZeneca and Bristol-Myers Squibb. "More and more we're using our technology for drug discovery," says Contag. "For instance, we have a whole series of animals that respond to toxicological stress. Or if drug metabolism is an issue, we have a series of animals that will tell you which enzyme is turned on."

Xenogen is now developing its technology for use with human subjects, and is also working on improving image quality. Although current resolutions cannot match those of PET or MRI, the technique can provide much more functional information. "There are things you can learn using optical imaging you cannot using PET and MRI," Contag says. "This is a desktop instrument, and that makes a huge difference as to where you apply it. It's very easy to use and the results are pretty spectacular." 
bears little resemblance to the traditional microscope. The user just has to add a monitor and a computer mouse.

"The Coolscope is basically a brightfield imaging system," says Robert Forster, general manager for Nikon Instruments UK. "It's primarily aimed at the clinical market for consultant pathologists to share images in multidisciplinary meetings with surgeons and radiologists. The Coolscope enables them to look at a patient's specimen on a microscope glass slide. It also enables them to view that slide remotely and to control the functions from anywhere in the world, providing you get the correct access through the hospital networks."

Digital technology is changing lab microscopes in many different ways. The DM DigitalMicroscope range produced by Leica Microsystems of Wetzlar, Germany, is not based on a digital camera as the name might imply, although Leica's DC range of cameras can be added as extras. Here, 'digital' refers to an integrated user-interface and control system.

"What scientists want is to concentrate on their work," says Michael Ganser, project leader for the Leica DM4000 B and DM5000 B microscopes for biological applications. "Ten or fifteen years ago, scientists loved their microscopes and knew exactly how to work them, but this has changed and microscopes are becoming more and more of a tool. What we tried to do is make it really easy to operate the microscope."

The DM series includes what Leica calls "intelligent automation" — push-button controls to rapidly configure the microscope for complex procedures. Automatic aperture and light adjustment, and colourintensity control allow the microscope to keep itself at the optimum settings. The DM4000 B and DM5000 B are mainly aimed at biomedical applications and pathology, and can be integrated with control systems for fluorescence, cytogenetics or other common techniques.

\section{Live cell imaging}

With the completion of the human genome sequence, the emphasis in biomedical research is now firmly back on functional biology and attempts to characterize and understand the proteome. To do this, researchers want to look inside live cells to study the expression and function of genes and proteins within particular pathways.

Using fluorescence to image living cells has become a major application for microscope producers and users. As well as its stand-alone microscopes, Leica Microsystems also produces the AS MDW, a dedicated workstation for the rapid imaging of molecular interactions in live cells. "All the hardware components, such as camera and piezo-focus, are designed in a way to optimize the speed of acquiring images," says Werner Kampe, marketing manager for Leica's compound-microscopy business unit. "That's important not just for scientists; it's also important not to damage the living cells under the microscope. The

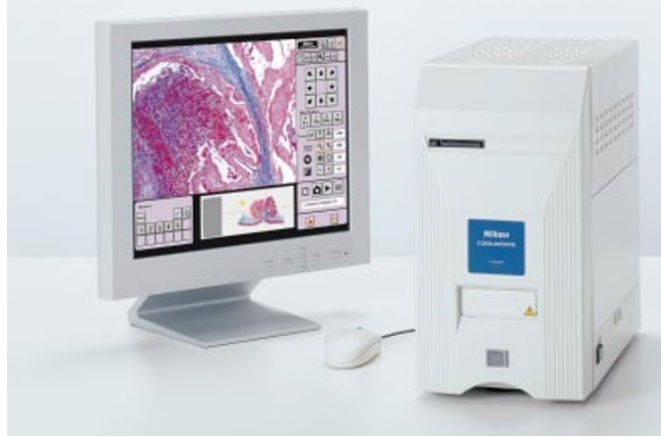

Easy viewing: Nikon's Coolscope.

longer they're under the light, the shorter their lifetime is." Sophisticated software means that cell interactions can be recorded in three dimensions, and merged into a time-lapse movie of changes over several days — something that Leica refers to as four-dimensional imaging.

A rival system from PerkinElmer of Wellesley, Massachusetts, boasts six-dimensional imaging of live-cell interactions. As well as three dimensions of space and one of time, the UltraView RS can image cellular interactions at two additional wavelengths. "To be able to do that for a live cell and get meaningful data you need to be sampling every few seconds," says Terry McCann, cellular sciences business manager at PerkinElmer. "UltraView RS takes advantage of the speed of the system so that we can generate these multidimensional images on biologically relevant timeframes."

\section{EASING THE STRAIN}

Many microscope users know all too well that prolonged use can be a real pain. Many systems demand that the user maintains a rigid body posture which can become increasingly uncomfortable, and concentrating on the magnified image for long periods can lead to eye fatigue and nausea. It can all add up to a serious reduction in accuracy and efficiency in the short term, and a long-term risk of debilitating repetitive strain injury (RSI).

Many manufacturers now design their microscopes to be ergonomically efficient, making operation as simple and intuitive as possible while

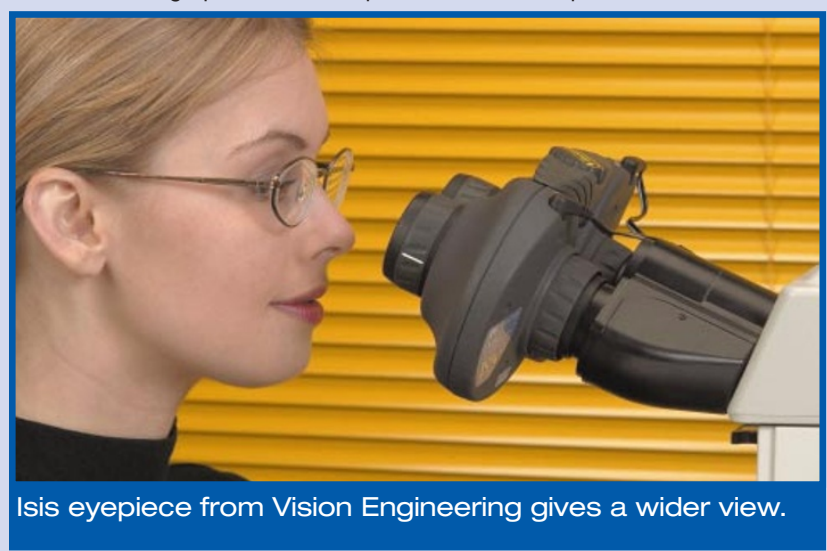

minimizing the risk of strain or fatigue. Leica Microsystems of Wetzlar in Germany, for example, worked with the Fraunhofer Institute of Stuttgart, Germany, to design its DM series of microscopes. The main control panels are positioned for direct and intuitive access, and height, viewing angle and eyepiece length can be adjusted to suit the user.

The narrow field of view from most microscope eyepieces is a major cause of eye strain and bad posture. Users who wear spectacles often have to remove them, increasing the risk of eye strain; and many users also suffer the distraction of floating fragments of tissue debris in the eye.

Vision Engineering of Woking, UK, a company specializing in microscope ergonomics, claims to have solved these problems with an eyepiece called the Isis expanded-pupil system. Fitted in place of the traditional eyepiece, the Isis is based on a small disc containing more than 2 million microlenses. This disc rotates at about 5,000 r.p.m., so that the millions of individual optical paths are merged to form a single high-clarity image, with an exit field around ten times as wide as from a conventional eyepiece.

"The key thing is the image, and where the image leaves the microscope and meets the operator," says international marketing manager Geoff Collins. "Our technology allows the image to have a much wider exit pupil, which means you can see it from much further away, from a greater range of angles. That allows you to move your head around, wear spectacles, and be further away from the eyepiece." Isis is available on Vision's own range of laboratory microscopes, and as an accessory to replace the conventional eyepieces on a wide range of commercial microscopes. 
The UltraView system is based on confocal microscopy, an optical sectioning technique that uses a scanning laser to produce high-resolution images from the fluorescence emitted by the sample. Traditional confocal microscopes can be too slow to study the most rapid cell processes, and the intensity of laser light can damage living cells.

Rather than using a single pinpoint laser beam, UltraView uses a broad beam imaged 兽 through two rapidly rotating discs: one, called a Nipkow disc, contains several thousand pinpoint holes; the other is an aligned disc featuring an equal number of microlenses to focus light onto the former. "You end up with 1,000 laser beams which shower the sample with light," says McCann. "Instead of one point being imaged, you have 1,000 points being imaged. You can see the whole field of view more quickly, and you also use very much less light."

\section{High-throughput scanning}

Live-cell imaging is also being taken into the high-throughput environment of industrial drug screening. The combination of high-throughput screening and highcontent imaging of cells was pioneered by Pittsburgh-based Cellomics (see 'High throughput goes 3D'), and the major producers of automated lab equipment are now moving into the field.

The IN Cell Analyzer 3000 produced by Amersham Biosciences of Buckinghamshire, UK, is a confocal imaging system designed to perform high-resolution cellular

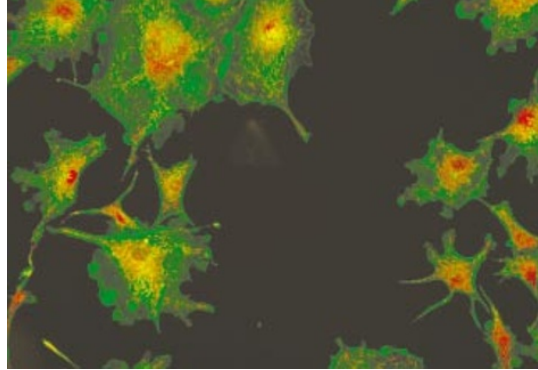

ImageXpress: neural stem cells.

assays at the rates demanded by today's automated drug-discovery labs.

"There's an increasing need to understand what's going on within the cell, and to do that in the environment of highthroughput screening is extremely valuable," says John Anson, vice-president for bioassay development at Amersham. "The objectives and a lot of the resolution come from existing technology, but what we've been able to do is build in the robustness and speed to take this through to the highthroughput environment."

Although the system uses laser-based autofocusing and other technologies to ensure rapid imaging of its well plates, the key to high throughput is in the software. "What we've been able to do is develop a software regime with the capability and speed not only to capture the data but to analyse it in a very short space of time," Anson says. "In the time it takes to mechanically move from one cell to the next, the software is doing all the work to generate knowledge from the image it's just taken." Integrated software also allows data to be combined with data from other Amersham systems such as its Typhoon and Storm series of gel scanners. "This technology isn't necessarily stand-alone," notes Anson. "Everyone would be interested in comparing data from cellular assays with in vitro assays such as gene expression in gel."

Integrated software is also at the heart of the high-throughput capabilities of the ImageXpress system produced by Axon Instruments of Union City, California. Like Amersham's IN Cell, ImageXpress is a rapid, robust automated system for imaging and analysing live cells, with applications in target evaluation, compound optimization and toxicology tests. The system incorporates a general-purpose analysis engine that can be applied to many different assays through a toolbox of dedicated software. Axon has recently developed sophisticated neuronal image-analysis software in association with the Australian research body CSIRO, which is now included in ImageXpress's toolbox.

Automated operation and image processing are based on the common VB scripting protocol, allowing ImageXpress to be integrated with plate-loading robots and other automated systems. Axon's Acuity image-analysis software, meanwhile, allows the import of data from other systems for multiparametric data mining.

"I think this technology is reaching a consolidation phase where the capabilities of the

\section{HIGH THROUGHPUT GOES 3D}

High-throughput analysis of cellular processes is now entering the mainstream for drug discovery, but Pittsburgh-based Cellomics has been working in this area for some time. Founded in 1996 to focus on the automated analysis of cell arrays, Cellomics offers an integrated package of imaging platforms with full environmental control, bioassays and bioinformatics and analysis software.

The firm produces two core platforms: KineticScan analyses cellular and intracellular interactions over time, and can deal with either live or fixed cells, whereas ArrayScan generates information-rich data on the effects of drug candidates on cells and is aimed at target validation and lead optimization applications.

The modular architecture of ArrayScan VTI, the fifth and latest generation of the system, allows users to increase optical capabilities by adding filters and objectives, and software to handle and visualize data better. The system also addresses the two conflicting aims of array screening - the need for high throughput, and the need for information-rich, high-resolution data including three-dimensional imaging.

"It takes time to acquire various z-positions and use higher magnification, so throughput is at odds with 3D resolution," says Martin Pietila, highcontent screening instrumentation product manager at Cellomics. "For the first time we've been able to offer both operating modes."

The latest ArrayScan includes the ApoTome optical-sectioning device developed by Carl Zeiss Microscopy of Jena, Germany, which can be deployed as required. ApoTome allows confocal-style three-dimensional imaging with a conventional fluorescence microscope, by projecting the image of a grid structure into the focal plane of the specimen. Combining images with the grid in three positions within this plane produces an optical section with improved contrast and resolution in the z-axis.

"This affords scientists precise control of sampling applications," Pietila says. "It allows them to take multiple measurements of different $z$-positions to look for structures with variable distribution in three dimensions." The ArrayScan VTI can analyse up to 150 96-well plates a day using ApoTome, or 260 a day without.

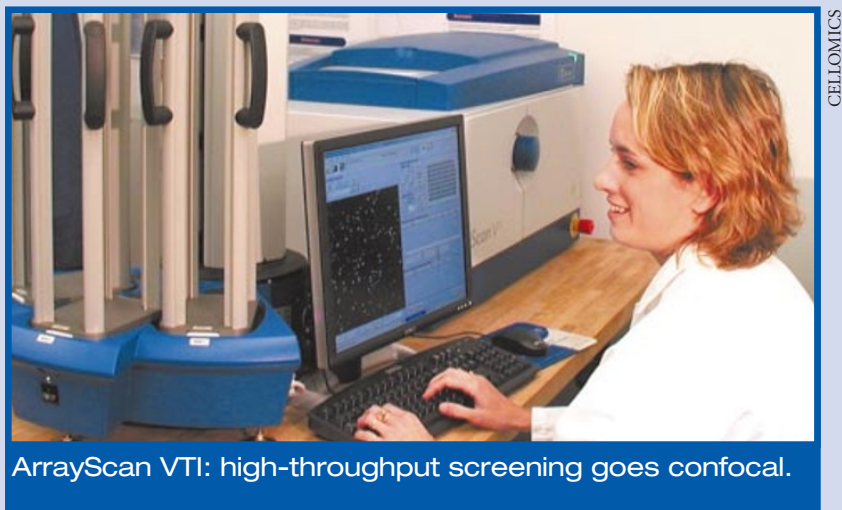


systems are starting to be appreciated and people are looking at what can be done with large amounts of data from these kinds of instruments - at multiparametric studies of cells and what kinds of coordinations you can derive between different families of cells," says Andrew Olson, product line manager for high-throughput cellular imaging at Axon.

\section{Electron microscopes}

Cell biologists are also turning to electron microscopy to understand supramolecular structures at the atomic level. Transmission electron microscopy (TEM) has long been used to visualize cell ultrastructure in plasticembedded sections of biological material, but it is now increasingly being used for three-dimensional imaging of whole cells, cell organelles and protein complexes.

To understand how protein complexes execute biological functions, researchers need to know the structures of these large, fragile constructions, and have deployed techniques such as nuclear magnetic resonance (NMR) spectroscopy, which offers only limited resolution, and X-ray crystallography, which requires large numbers of molecules in a crystalline array. TEM offers a more flexible solution.

"Over the past five years, the proof of concept has been given that TEM can be very instrumental in determining the threedimensional structure of these huge protein complexes," says Werner Hax, life-sciences business development manager at electron-

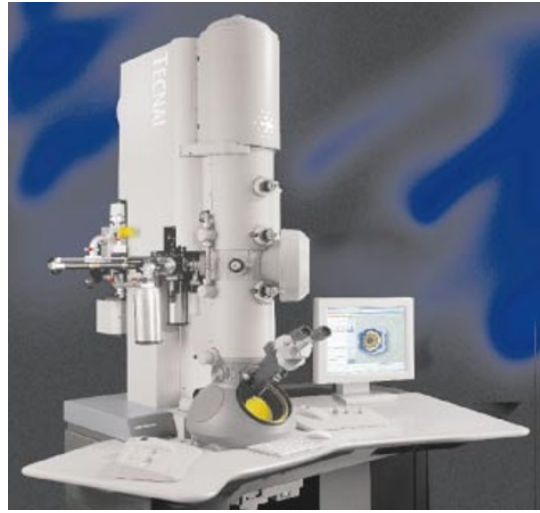

Tecnai G2 Polara from FEI.

microscope manufacturer FEI of Hillsboro, Oregon. "TEM can tell you exactly where in the cell these proteins and protein complexes are located, so you can bring everything into perspective."

The Tecnai G2 Polara from FEI is a TEM optimized for structural biology tomography and single-particle analysis. "Imaging of frozen hydrated biological samples within a TEM has to be done with the greatest care, as the electron beam can damage the object under investigation," says Hax. "The Tecnai G2 Polara allows observation of proteins at temperatures below $10 \mathrm{~K}$ and provides the other conditions to minimize beam damage."

The Tecnai instruments are fully digitally controlled, with accessories such as cameras and X-ray detectors embedded, allowing them to be integrated into auto- mated processes. "A lot of data have to be acquired and a lot of computational technology has to be applied to reconstruct the $3 \mathrm{D}$ representation of each protein in the cell," Hax says. "To do that within an acceptable period of time, particularly if you want to get this applied in industry in drug discovery, automation is an absolute must. Without that, TEM will never have the dominant role it can play."

Less specialized electron microscopes are finding wider use as an everyday tool for biological research. Delong Instruments of Brno, Czech Republic, has produced what it claims is the world's smallest TEM. The LVEM5 is intended primarily for biological and medical research, and provides highcontrast images of thin sections and particles such as viruses, enzymes, ribosomes, proteins and DNA, without the need for staining with heavy metals.

"This is practicable only by a considerable decrease of the energy of the imaging beam electrons," says Delong's Michal Drsticka. "When the energy is decreased roughly ten times, the contrast is ten times higher. The microscope therefore can be used both in university research and biological objects diagnostics."

The LVEM5 is around a tenth of the size of a conventional TEM, and could join the optical microscope as an everyday benchtop instrument. The bioscience researcher has never had such a choice of ways of seeing. Tim Chapman is a freelance technology journalist based in Halifax, UK.

\section{SCANNING THE SURFACE}

Photon tunnelling microscopy (PTM) is a unique surface-scanning technology that can produce real-time three-dimensional images at unparalleled resolution of biological samples, including live cells. The basic technology can be used with common optical microscopes, and can achieve better vertical resolution than a scanning electron microscope without the need for a scanning tip.

The system addresses a fundamental problem of optical imaging - the smaller an object is relative to the wavelength of incident light, the larger the angle of the diffracted light. For very small objects, the light is diffracted at such a large angle that it cannot leave the object, and is bound in an 'evanescent field', the intensity of which decays exponentially with distance. To see such a small object, you have to take the lens to within a few hundred nanometres of the object's surface without damaging either the instrument or the sample. And unless you operate in a 'clean room', you will have to deal with dust that is many times larger than this distance.

The technology that enables PTM was developed by John Guerra in his former role as senior principal engineer at Polaroid. In June 2002, Guerra launched his own company, Nanoptek, based in Concord, Massachusetts, to develop and commercialize the technology. The key to Nanoptek's approach is a square of soft, flexible polymer film, $75 \mathrm{~mm}$ across and 15-20 $\mu \mathrm{m}$ thick, called a transducer, which is applied over the sample. This is made optically continuous to the near-field lens with a fluid coupling. "This allows you to move around this 75-mm area very quickly without damaging the microscope or the sample," Guerra explains. The film also covers any dust particles on the sample.

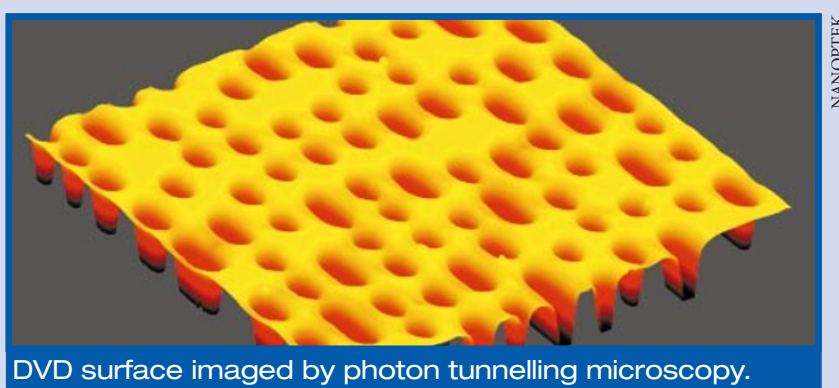

Over the 400-nm vertical range of the evanescent field, the coupling goes from total to zero, creating a grey-scale image in the microscope. A 12-bit CCD camera would give a vertical resolution of $0.1 \mathrm{~nm}$ - better than that achievable with scanning electron microscopy (SEM), and equal to that of atomic force microscopy (AFM), with the added advantage of real-time imaging.

"You can get $200-\mu \mathrm{m}$ fields of view at video rates. You can watch chemotaxis in progress or cells moving," says Guerra. At about $100 \mathrm{~nm}$, lateral resolution is not as good as with SEM or AFM, but is better than that of confocal or phase-shifted interference microscopy. Nanoptek is also planning to combine PTM with phase-shifting to increase the lateral resolution to $0.1 \mathrm{~nm}$. "That will be the next exciting development," Guerra says. "You will be able to image viruses and very, very small biological materials." T.c. 